\title{
Universality of the single-particle spectra of cuprate superconductors
}

\author{
Lijun Zhu, Vivek Aji, Arkady Shekhter and C. M. Varma \\ Department of Physics and Astronomy, University of California, Riverside, California 92521
}

\begin{abstract}
All the available data for the dispersion and linewidth of the single-particle spectra above the superconducting gap and the pseudogap in metallic cuprates for any doping has universal features. The linewidth is linear in energy below a scale $\omega_{c}$ and constant above. The cusp in the linewidth at $\omega_{c}$ mandates, due to causality, a "waterfall", i.e., a vertical feature in the dispersion. These features are predicted by a recent microscopic theory. We find that all data can be quantitatively fitted by the theory with a coupling constant $\lambda_{0}$ and an upper cutoff at $\omega_{c}$ which vary by less than $50 \%$ among the different cuprates and for varying dopings. The microscopic theory also gives these values to within factors of $O(2)$.

PACS numbers: 74.20.Mn, 74.25.Jb, 74.72.-h
\end{abstract}

Introduction. With increased refinement of technique and imaginative use, angle-resolved photoemission spectroscopy (ARPES) on the high temperature superconductors has revealed that novel physical principles determine the single-particle spectra in such compounds [1, 2]. Recently, the single-particle spectra over an energy range from the chemical potential to about $1 \mathrm{eV}$ have been deduced for various metallic dopings in different cuprates $[3,4,4,5,6,7,6,6,9]$. Also, recently a microscopic theory [11] has been formulated which derives the fluctuations leading to the phenomenological marginal Fermi liquid(MFL) [12] and their coupling to fermions. MFL had previously been tested only for low energies and near optimal doping in Bi2212 [13, 14, 15] with adjustable couplings. Here we test crucial new features of the microscopic theory including its universality, its cut-off and coupling functions, by comparing with recent ARPES data in 4 different cuprate families and at different dopings.

The most important results of these recent ARPES experiments may be summarized as follows:

(i) The spectra for energies $\omega$ in the range of interest (above the superconducting gap and the pseudogap energy scales) are universal; they have the same functional form for all cuprates and for all metallic dopings. Moreover, even the parameters in the functional form vary less than by a factor of 2 over the entire range of cuprates for which data is available, irrespective of whether they are underdoped(UD), optimally doped(OP) or overdoped(OD).

(ii) The momentum distribution curves (MDC) at constant energy $\omega$ is a Lorentzian with width $w_{\mathbf{k}}(\omega)$. In the energy range of interest $w_{\mathbf{k}}$ varies linearly with $\omega$ up to a cutoff above which it is approximately a constant. This is modified if the bare velocity $\mathbf{v}(\mathbf{k})$ varies within $w_{\mathbf{k}}$, which happens as the bottom of the band is approached. See Fig. 3 below for representative experimental data.

(iii) The peak of the MDC as a function of $\omega$ moves with $\mathbf{k}$ defining the renormalized dispersion $\varepsilon(\mathbf{k})$. The observed dispersion $\varepsilon(\mathbf{k})$ follows the band structure $\epsilon_{\mathbf{k}}$ with a smooth renormalization factor up to $\omega \approx E_{1}$.
Above $E_{1}$, the "velocity" $d \varepsilon(\mathbf{k}) / d k$ sharply increases up to another cutoff $E_{2}$ where $\varepsilon(\mathbf{k})$ resumes the normal dispersion. The nearly vertical dispersion has been picturesquely termed a "waterfall" [3]. In the energy range, $E_{1} \lesssim \omega \lesssim E_{2}$, there is also an indication of multiple $\varepsilon(\mathbf{k})$ for fixed $\omega[6,8]$. $E_{1}$ varies systematically being largest in the $(\pi, \pi)$ direction and smallest in the $(\pi, 0)$ direction 8]. Similarly, the position of the "waterfall" in k-space varies systematically.

All these features follow quantitatively from the quantum-critical fluctuations derived recently [1]. We find that, given the bare band structure $\epsilon_{\mathbf{k}}$, all available data can be fitted with the two parameters of this theory, a sharp cutoff $\omega_{c}$ and a coupling constant $\lambda_{0}$ calculable to factors of $O(2)$.

Single-particle spectral function. The single-particle spectral function deduced by ARPES is given

$$
A(\mathbf{k}, \omega)=\frac{-\operatorname{Im} \Sigma(\omega, \mathbf{k}) / \pi}{\left[\omega-\operatorname{Re} \Sigma(\omega, \mathbf{k})-\epsilon_{\mathbf{k}}\right]^{2}+[\operatorname{Im} \Sigma(\omega, \mathbf{k})]^{2}},
$$

where $\Sigma(\omega, \mathbf{k})$ is the self-energy function. The band structure $\epsilon_{\mathbf{k}}$ is in general fitted by the tight-binding dispersion[16].

Microscopic Theory. A microscopic theory for the cuprates 11, 17, 18] is based on the realization, that the central organizing feature in the physics of the metallic phase of the cuprates are quantum critical fluctuations of loop currents. In this theory the ordering of these loop currents below $T_{g}$ (PG in inset of Fig. 11) leads to a phase which breaks time-reversal symmetry but preserves translational symmetry. Direct evidences for such an ordered state have been obtained by polarized neutron scattering in $\mathrm{YBa}_{2} \mathrm{Cu}_{3} \mathrm{O}_{6+x}$ [19] and by dichroic ARPES experiments in $\mathrm{Bi}_{2} \mathrm{Sr}_{2} \mathrm{CaCu}_{2} \mathrm{O}_{8+x}$ [20], for various $x$ in the pseudo-gap phase. The properties in the entire funnel shaped region (I) in inset of Fig. 1 are determined by the quantum critical fluctuations of the loop currents. Therefore universal properties are predicted for $\omega$ larger than the superconducting gap or the pseudogap for all $x$ in the metallic phases on either side of $x_{c}$. 
The microscopic theory of the quantum-critical fluctuations [11] gives their absorptive part to be

$$
\begin{array}{cl}
\operatorname{Im} \chi(\mathbf{q}, \nu)=-\chi_{0} \tanh \frac{\nu}{2 T}, & |\nu|<\omega_{c} \\
0, & |\nu|>\omega_{c} .
\end{array}
$$

$\omega_{c}$ is a cutoff and $\chi_{0}$ gives the integrated weight of the fluctuations. In the microscopic theory [11], $\omega_{c}^{2} \approx$ $2 E V R^{2}$, where $E$ is the local repulsion or charging energy parameter, $V$ the nearest neighbor $\mathrm{Cu}-\mathrm{O}$ interaction, and $R$ is the dimensionless loop-current order parameter. $\chi_{0} \approx R / \omega_{c}$. For $E \simeq 5 e V, V \simeq 1-2 \mathrm{eV}$ and $R \simeq 0.1$ which is consistent with the neutron measurements, we expect $\omega_{c} \approx 0.3-0.5 \mathrm{eV}$.

Calculation of the Self-energy. The loop current fluctuations scatter fermions from $\mathbf{k}$ to $\mathbf{k}^{\prime}$ with the amplitude $\gamma\left(\mathbf{k}, \mathbf{k}^{\prime}\right)$. From the microscopic model, we find [21]

$\gamma\left(\mathbf{k}, \mathbf{k}^{\prime}\right)= \pm i \frac{V}{2}\left(s_{x}\left(k+k^{\prime}\right) s_{y}\left(k-k^{\prime}\right)-x \leftrightarrow y\right) S_{x y}\left(k, k^{\prime}\right)$,

where $s_{x, y}(k) \equiv \sin \left(k_{x, y} a / 2\right), s_{x y}(k) \equiv \sqrt{s_{x}^{2}(k)+s_{y}^{2}(k)}$, $S_{x y}\left(k, k^{\prime}\right) \equiv\left(s_{x y}^{-1}(k)+s_{x y}^{-1}\left(k^{\prime}\right)\right)$. The leading self-energy contribution is

$\Sigma\left(i \omega_{n}, \mathbf{k}\right)=T \sum_{\mathbf{q}, i \nu_{n}}|\gamma(\mathbf{k}, \mathbf{k}+\mathbf{q})|^{2} G\left(i \omega_{n}+i \nu_{n}, \mathbf{k}+\mathbf{q}\right) \chi\left(\mathbf{q}, i \nu_{n}\right)$,

where $\omega_{n}, \nu_{n}$ are Matsubara frequencies of the quasiparticle and the fluctuating mode, respectively. Given a q-independent $\chi$ and $\gamma\left(\mathbf{k}, \mathbf{k}^{\prime}\right)$ of the form of Eq. (3), the self-energy variation with $\mathbf{k}$ on a Fermi surface comes only from the separable $s$-wave part of $\left|\gamma\left(\mathbf{k}, \mathbf{k}^{\prime}\right)\right|^{2}$ which is $\propto\left(1-\cos k_{x} a \cos k_{y} a\right)\left(k \rightarrow k^{\prime}\right)$. This gives $\lambda_{k} \propto(1-$ $\left.\cos k_{x} a \cos k_{y} a\right)$ which varies by about a factor of 2 from the $(\pi, \pi)$ to the $(\pi, 0)$ directions for the Fermi surface of Bi2212 near optimal doping.

At $T=0$ the self-energy is easily evaluated to be

$$
\begin{aligned}
\operatorname{Im} \Sigma(\omega, \mathbf{k})= & -\lambda(\mathbf{k}) \frac{\pi}{2} \begin{cases}|\omega|, & |\omega|<\omega_{c} \\
\omega_{c}, & |\omega|>\omega_{c}\end{cases} \\
\operatorname{Re} \Sigma(\omega, \mathbf{k})= & -\frac{\lambda(\mathbf{k})}{2}\left[\omega \ln \frac{\omega_{c}}{|\omega|}+\left(\omega-\omega_{c}\right) \ln \frac{\left|\omega-\omega_{c}\right|}{\omega_{c}}\right. \\
& -(\omega \rightarrow-\omega)],
\end{aligned}
$$

where $\lambda(\mathbf{k})=\lambda_{0}\left\langle\gamma^{2}\right\rangle_{k^{\prime}} ; \lambda_{0}=N(0)\left(V^{2} / 4\right) \chi_{0}$ and $\left\langle\gamma^{2}\right\rangle_{k^{\prime}}$ is the average of $\left|\gamma\left(\mathbf{k}, \mathbf{k}^{\prime}\right)\right|^{2}$ over $\mathbf{k}^{\prime}$ on the Fermi surface. For the density of states per one spin species $N(0) \approx 1(\mathrm{eV})^{-1}$ and other parameters used above, we expect $\lambda_{0} \approx 1$.

Given such a weakly momentum dependent self-energy, the vertex corrections 22] to the self-energy are only of $O\left(\lambda \omega_{c} / W\right)$, where $W$ is the bare bandwidth of the conduction band. Using the $\omega_{c}$ and $\lambda$ fitted to the experiments, this ratio is of $O(0.1)$. The remaining processes, repeated scattering (self-consistent Born approximation)

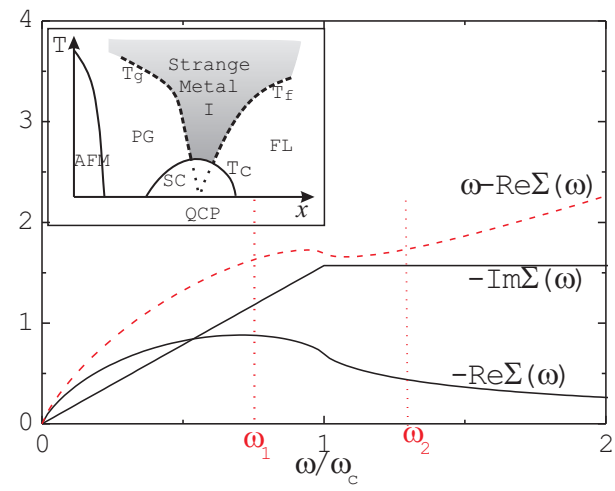

FIG. 1: The self-energy, and $\omega-\operatorname{Re} \Sigma(\omega)$ as functions of $\omega$ for $\lambda_{0}=1$. All quantities are dimensionless in units of $\omega_{c}$. The observable dispersion $\varepsilon(\mathbf{k})$ for a given bare $\epsilon_{\mathbf{k}}$, solved by Eq. (6) is equivalent to the intersection of $\omega-\operatorname{Re} \Sigma(\omega)$ with a horizontal line at $\epsilon_{\mathbf{k}}$. As $\omega-\operatorname{Re} \Sigma(\omega)$ has a wide reentrant region for $\omega_{1} \leq \omega \leq \omega_{2}$, the observed dispersion falls from $\omega_{1}$ to $\omega_{2}$ for a very small variation in $\epsilon_{\mathbf{k}}$ producing the "waterfall". Insert shows the phase-diagram of the cuprates.

produce no singular corrections. At low energies compared to $\omega_{c}$, Eq. (5) reduces to the MFL form deduced earlier [12], except for the weak momentum dependence.

Given the sharp change of the slope in the imaginary part near $\omega_{c}$, the real part has a logarithmic divergence in its slope at $\omega_{c}$ before changing from its low energy form $\propto \omega \log |\omega|$ to $1 / \omega$ for $\omega \gg \omega_{c}$. This sharp variation of $\operatorname{Re} \Sigma(\omega)$ near $\omega_{c}$ is responsible for the observed "waterfall" feature as we now proceed to show.

The Waterfall. The dispersion of the quasi-particles, $\varepsilon(\mathbf{k})$ given by

$$
\varepsilon(\mathbf{k})-\operatorname{Re} \Sigma(\varepsilon(\mathbf{k}))-\epsilon_{\mathbf{k}}=0 .
$$

As shown in Fig. 1 $\omega-\operatorname{Re} \Sigma(\omega)$ has a wide reentrant region from $\omega_{1} \leq \omega \leq \omega_{2}$. The solution of Eq. (6) therefore produces a "waterfall" in the dispersion $\varepsilon(\mathbf{k})$ because it varies over the large energy range $\omega_{1}$ to $\omega_{2}$ for a very small variation in $\mathbf{k}$. The multiple solutions obtained in this region are within $\operatorname{Im} \Sigma(\omega)$ for $\lambda$ of $\mathrm{O}(1)$. Above $\omega \simeq \omega_{2}$, the dispersion becomes just a renormalized band structure. The calculated "waterfall" is shown in Fig. 2. The spectral intensity maps in Fig.2(d-f) should be compared with Fig. 1(a-c) of Ref. [8].

Comparison with Experiments: The calculated selfenergy at $\lambda_{0}=1$ (suitable to fit the experimental data 8] for $\left.\mathrm{La}_{1.83} \mathrm{Sr}_{0.17} \mathrm{CuO}_{4}\right)$ is shown in Fig. 11 The experimental MDC width for this compound and the calculated widths for three different cuts are compared with experiment in Fig 3 In Fig $4(a)-(c)$, we compare the experiments [3] for the dispersion of three Bi2212 samples at different dopings with calculations with $\omega_{c}=0.5, \lambda_{0} \sim 1$. In Fig 4 (d), we compare the measured linewidth for an UD-LSCO sample, an OP-Bi2201 sample and an OPBi2212 sample with calculations with parameters given 


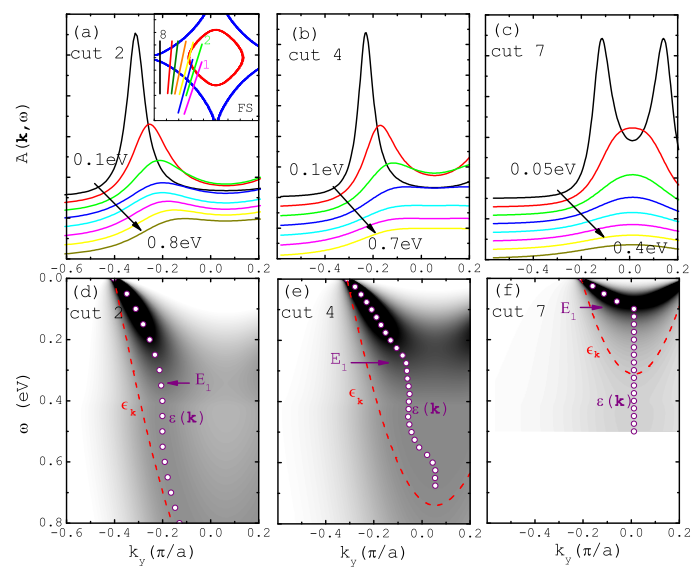

FIG. 2: (a),(b) and (c): calculated MDCs for three of the momentum cuts 2,4 and 7 shown in the inset to Fig. (a) for which data is available from Ref. 8 . The MDC's are shown at various energies $\omega$ labeled in the figures. Figs. (d),(e) and (f) are spectral intensity maps, for the same cuts correspondingly. From the intensity maps, we can identify the dispersion $\varepsilon(\mathbf{k})$ marked by circles; the bare dispersion $\epsilon_{\mathbf{k}}$ are shown in dashed lines. The inset of Fig. (a) shows the Fermi surface and eight momentum cuts done in experiments [8]. It also shows the positions where the "waterfall" are expected in $\mathbf{k}$-space for radial cuts with the additional contour drawn inside the Fermi surface.

in the figure caption.

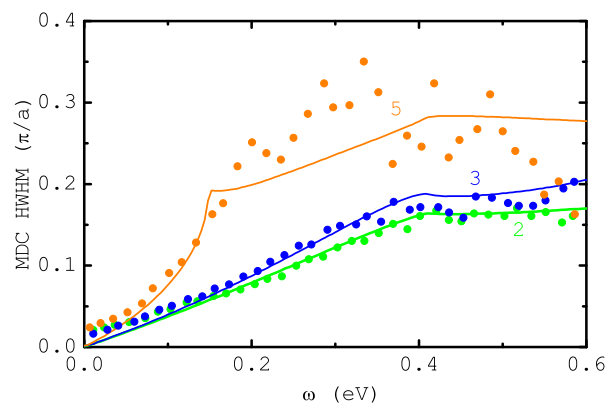

FIG. 3: The MDC half-width at half-maximum $w_{\mathbf{k}}(\omega)$ is shown for the cuts 2,3 and 5 of the inset of Fig. 2(a). The experimental data for the same cuts from Fig. 2 of Ref. 8 is also shown. Note that the experiments quote are done with an energy resolution of $30 \mathrm{meV}$, which accounts for the deviation from the theory at low energies. Higher resolution data [15] confined to lower energies is consistent with the theory.

Universality of the Data: The data and the comparison with experiments in Fig 3 and Fig. 4(a)-(d) attest to the universality of the single-particle spectra of the cuprates and of the quantitative success of the theory. Now we consider in detail each of the points (i) to (iii) of the experimental data and explain them successively.

(i) The physical properties in any quantum critical regime are universal, controlled by the scale-invariant critical fluctuations. Specifically, for $\omega$ larger than the su-
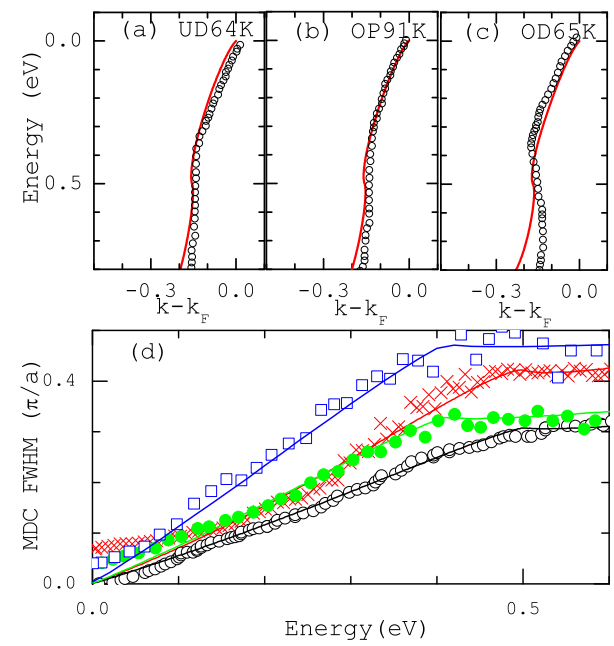

FIG. 4: Comparison between experimental and theory results (represented by symbols and lines, respectively) for various cuprate samples. (a)-(c) are calculated dispersions for three Pb-doped Bi2212 samples along the nodal cuts: UD with $T_{c}=$ $64 K$, OP with $T_{c}=91 K$ and OD with $T_{c}=65 K$. The experimental data shown are extracted from Fig. 1 of Ref. [3]. The tight-binding fitting parameters of the band structure are taken from Ref. 23]. All these samples are fitted by the parameters $\omega_{c}=0.5 \mathrm{eV}$ (for all) and $\lambda_{0}=0.98,1.01$, and 1.05, respectively. (d) shows the MDC linewidths (full width at half maximum) for different cuprate samples. $\circ, \times, \bullet$ and $\square$ represent OP-Bi2201 (nodal cut, Ref.[9]), OP-Bi2212 (nodal cut, Ref. [24]), LSCO 0.17 (cut 2 in Fig. 2 of Ref. [8]) and LSCO 0.145 (cut 1 in Fig. 3 of Ref. 25]), respectively. The corresponding theory fitting parameters are: $\lambda_{0}=0.99, \omega_{c}=$ $0.5 \mathrm{eV} ; \lambda_{0}=1.01, \omega_{c}=0.5 \mathrm{eV} ; \lambda_{0}=1.09, \omega_{c}=0.41 \mathrm{eV}$ and $\lambda_{0}=$ $1.64, \omega_{c}=0.41 \mathrm{eV}$.

perconducting gap or the pseudogap the self-energy is of MFL form and given in terms of only the two parameters $\omega_{c}, \lambda_{0}$ for each compound for all $x$. Weak dependencies in these parameters from variation in microscopic parameters due to varying $x$ or $T$ may occur of course. We find however that for a given compound, a single value of these parameters is adequate to fit all the available data for different $x$ and for all momentum directions.

It is worth noting that the spectra for energies below the pseudogap energy and $T \leq T_{g}$ is also scale-invariant with a new scale $\propto T_{g}(x)$ [26, 27].

(ii) Suppose at certain energy $\omega$, Eq. (6) is satisfied for $\mathbf{k}=\mathbf{k}_{0}$. Since the self-energy does not depend significantly on $\mathbf{k}$, we can expand the spectral function in $\left(\mathbf{k}-\mathbf{k}_{0}\right)$. The MDC is then a Lorentzian with width $w_{\mathbf{k}}$ given by $\operatorname{Im} \Sigma(\omega) / v\left(\mathbf{k}_{0}\right)$ where $v\left(\mathbf{k}_{0}\right)=$ $v_{y}\left(\mathbf{k}_{0}\right)+v_{x}\left(\mathbf{k}_{0}\right)\left(k_{x}-k_{x 0}\right) /\left(k_{y}-k_{y 0}\right)$, is the bare velocity in the momentum-cut direction. This expansion also requires that within $\left(\mathbf{k}-\mathbf{k}_{0}\right) \approx w_{\mathbf{k}}$, the velocity $\mathbf{v}_{\mathbf{k}}$ is nearly a constant.

As discussed above $\operatorname{Im} \Sigma(\omega)$ increases linearly in $\omega$ for $\omega \lesssim \omega_{c}$ and is constant beyond. Therefore if $\mathbf{v}_{0}(\mathbf{k})$ varies slowly with $\mathbf{k}$ as in cut 2 in Fig. 2. MDC linewidths also 
vary linearly in $\omega$, i.e., $w_{\mathbf{k}} \propto \omega$. Away from the nodal momentum directions, $\mathbf{v}_{0}(\mathbf{k})$ varies considerably as in cut 4 and higher of Fig. 2. As a result, MDCs' linewidth deviates from the linear- $\omega$ dependence. This accounts for the MDC width of cut 5 shown as an example in Fig. 3 and the higher cuts. If the MDC linewidth is multiplied by the bare velocity at each $\mathbf{k}$ in any direction, a linear dependence of the width with $\omega$ is obtained both in theory and the experiments.

(iii) Comparing Figs. 2(d-f), we can see that there are two distinct reasons for the "waterfalls". If $\epsilon_{\mathbf{k}}$ reaches $\omega_{1}-\operatorname{Re} \Sigma\left(\omega_{1}\right)$ at $\mathbf{k} \approx \mathbf{k}_{0}$ as $\mathbf{k}$ is varied along the momentum cut, e.g., cut 2 in Fig. 2, $\varepsilon(\mathbf{k})$ follows the "waterfall" between $\omega_{1}$ and $\omega_{2}$, which correspond to $E_{1}$ and $E_{2}$ defined in experiments.

If the momentum cuts are sufficiently away from the nodal cut such that the bottom of the band is very shallow, $\epsilon_{\mathbf{k}}$ never reaches $\omega_{1}-\operatorname{Re} \Sigma\left(\omega_{1}\right)$; e.g., cuts $5-8$ in Fig. 2. The observed dispersion $\varepsilon(\mathbf{k})$ then follows Eq. (6) to its maximum value at the bottom of the band $\mathbf{k}_{m}$. For higher energies, there are no solutions to Eq. (6). In this case the MDC curves stay centered at $\mathbf{k}_{m}$ which leads to another type of "waterfall". $E_{1}$ in this case is nearly the energy of the bottom of the renormalized band, and gets continuously smaller as the bottom of the band (where the velocity is zero) becomes continuously more shallow from the $(\pi, \pi)$ to the $(\pi, 0)$ direction. The variation of the position of the "waterfall"s, Fig. 3 of Ref. [8] and Fig. 3 of Ref. [6] is thereby explained. In addition, the linewidth is no longer given by $\operatorname{Im} \Sigma / v_{0}$, leading to an additional cusp in linewidth at $E_{1}$ (e.g., cut 5 in Fig. 3 ).

However, if radial cuts are taken to avoid the shallow band, the position of the "waterfall" in momentum space is always the locus of $\mathbf{k}$ where $\varepsilon(\mathbf{k}) \approx \omega_{1}$. This locus is shown in Fig. 2(a) for radial cuts and is to be compared with data in Fig. 4 of Ref. [8] and Fig. 4 of Ref. [6].

Concluding remarks. The experimental results discussed place strong constraints on a theory applicable to the cuprates. Specifically, the experiments give a scattering rate linear in $\omega$ up to a sharp cut-off at $\omega_{c}$ and constant above with a coefficient which is a weak function of $\mathbf{k}$. This behavior is found in the entire 'strange metal' region of the phase diagram. We do not know any ideas proposed for cuprates besides those discussed here which give these properties.

In this paper, we have pointed out the universal aspects of the measured single-particle self-energy in cuprates and shown that its functional form and even its magnitude is consistent with the recent microscopic theory of quantum critical fluctuations 11]. These fluctuations are predicated on the existence of an unusual symmetry breaking in underdoped cuprates for which considerable experimental evidence has also been adduced.

Acknowledgments. $\mathrm{CMV}$ is especially grateful to J. Mesot for interesting him in the problem and to him, J. Chang, S. Paillhès and C. Mudry for a detailed discussion of the data. Thanks are also due to J. Graf and A. Lanzara for communications regarding their data.

[1] J. C. Campuzano, M. R. Norman, and M. Randeria, in The Physics of Superconductors Vol. II, ed. K. H. Bennemann and J. B. Ketterson (Springer, Berlin, 2004), p. 167.

[2] A. Damascelli, Z. Hussain, and Z.-X. Shen, Rev. Mod. Phys. 75, 473 (2003).

[3] J. Graf et al., Phys. Rev. Lett. 98, 067004 (2007).

[4] B. P. Xie et al., Phys. Rev. Lett. 98, 147001 (2007).

[5] T. Valla et al., cond-mat/0610249.

[6] J. Graf, G.-H. Gweon, A. Lanzara, cond-mat/0610313

[7] Z.-H. Pan et al., cond-mat/0610442

[8] J. Chang et al., Phys. Rev. B 75, 224508 (2007).

[9] W. Meevasana et al., Phys. Rev. B 75, 174506 (2007).

[10] In D.S. Inosov et al., cond-mat/0703223, it is pointed out that the variation of the photoemission matrix elements with momentum can lead to misleading conclusions about the spectral function and produce faux "waterfalls". To avoid this one should only take seriously the spectral function deduced from the experiment which obey Kramers-Kronig relations.

[11] V. Aji and C. M. Varma, Phys. Rev. Lett. 99, 067003 (2007).

[12] C. M. Varma et al., Phys. Rev. Lett. 63, 1996 (1989).

[13] T. Valla et al., Science 285, 210 (1999).

[14] E. Abrahams and C. M. Varma, Proc. Natl. Acad. Sci. USA 97, 5714 (2000).

[15] A. Kaminski et al., Phys. Rev. B 71, 014517 (2005).

[16] The band structure $\epsilon_{\mathbf{k}}=-2 t\left(\cos k_{x} a+\cos k_{y} a\right)-$ $4 t^{\prime} \cos k_{x} a \cos k_{y} a-2 t^{\prime \prime}\left(\cos 2 k_{x} a+\cos 2 k_{y} a\right)-\mu$ was used in Ref. [8] to fit the Fermi surface of LSCO, with $t^{\prime} / t=$ $-0.144, t^{\prime \prime} / t=0.072$, and $\mu / t=-0.84$. We use the same values with $t=0.48 \mathrm{eV}$ consistent with fits to LDA calculations, see, e.g., R. J. Radtke and M. R. Norman, Phys. Rev. B 50, 9554 (1994).

[17] C. M. Varma, Phys. Rev. Lett. 83, 3538 (1999).

[18] C. M. Varma, Phys. Rev. B. 73, 155113 (2006).

[19] B. Fauqué et al., Phys. Rev. Lett. 96, 197001 (2006).

[20] A. Kaminski et al., Nature (London) 416, 610 (2002).

[21] This is a straightforward extension to $\mathbf{q} \neq 0$ of the $\mathbf{q}=0$ result of Eq. (44) in Ref. 18].

[22] A. B. Migdal, Zh. Eksp. Teor. Fiz. 341438 (1958) [Sov. Phys. JETP, 7996 (1958)].

[23] A. A. Kordyuk et al., Phys. Rev. B 67, 064504(2003).

[24] J. Graf and A. Lanzara, private communication.

[25] J. Chang et al., arXiv:0708.2782.

[26] A. Kanigel et al., Nature Physics 2, 447 (2006).

[27] C. M. Varma and L. Zhu, Phys. Rev. Lett. 98, 177004 (2007). 